\title{
Modular Design of DC-DC Converters for EV battery fast-charging
}

\author{
Rómulo Antão, Tiago Gonçalves, Rui Escadas Martins \\ Department of Electronics, Telecommunications and Informatics \\ Universidade de Aveiro \\ Campus of Santiago , 3810-193 Aveiro (Portugal) \\ Phone:+351 234370 355, e-mail: romuloantao@ua.pt
}

\begin{abstract}
.
Electric vehicles (EV) are emerging as a viable and environment-friendly solution for the daily commute. However, for a broader utilization of the EV, their autonomy range has to be increased. A well dispersed charging network and a fast and efficient battery charger system becomes one important factor for the success of the EV. This paper addresses the challenges of developing such High Power chargers and proposes a modular, easily scalable and efficient implementation of a switch-mode DC-DC converter for performing the constant-current/ constantvoltage battery charging process.
\end{abstract}

\section{Key words}

Electric vehicles charging system, switch-mode DC-DC converters, Forward Topology

\section{Introduction}

The high volatility of the crude oil price and the climate changes are one of the major concerns of nowadays global society. The high dependency of the economy in oil resources and near future reserves depletion brought great investment on alternative and cleaner energy sources. In recent years, the automotive industry grew particular interest on the development of electric vehicles (EV), mainly due to the higher efficiency of electric motors, cheaper electricity prices and the reduction of green-house gas emissions that cars directly produce.

Nowadays, plug-in hybrid electric vehicles (PHEV) are the approach adopted by many automobile manufacturers. The existing PHEV's were designed aiming an all-electric range compatible to the daily commuter requirements. They have small capacity batteries, typically $5 \mathrm{kWh}$ and can be completely charged typically in a $1 \mathrm{~h} 30 \mathrm{~m}$ period using the regular electrical outlet of $230 \mathrm{~V} / 16 \mathrm{~A}$. This charge time is perfectly compatible with an employee daily work schedule. The challenge poses, however, on the current generation of EV, which rely their autonomy totally on the battery capacity. For an increased market penetration, the range anxiety of the drivers is the chief concern to overcome. The new Nissan Leaf, one of the 2011 EV models, took a leap ahead, by integrating a 24 kWh battery offering a reported $160 \mathrm{Km}$ autonomy, a concept which has been more recently followed by
Opel/Chevrolet, Mitsubishi, Renault and others. However, the state-of-the-art of battery technology still poses big constrains in their power density, charging current, number of charging cycles and ultimately their cost. The lithium-ion batteries currently used combined with a high power charger (under constant current control) allow to achieve $80 \%$ of the maximum battery capacity of a vehicle like Nissan Leaf in less than 30 minutes (what still limits the usage of EV for longer distance trips).

Until further advances are obtained in the battery research area, charging infrastructures have to be broadly available. According to the developments recently unveiled by the SAE International J1772 committee [1], the battery charging systems can be classified in terms of their power level, and consequently their charging time. Considering the systems with higher power ratings, commonly known as DC fast charging systems, their specifications are following presented:

- DC Level I - The car battery is charged directly by a off-board DC charging infrastructure, being supplied a DC voltage (between $200 \mathrm{~V}$ and $500 \mathrm{~V}$ ) with a current up to $80 \mathrm{~A}$, with a maximum power level up to $40 \mathrm{KW}$.

- $\quad$ DC Level II - The car battery is charged directly by a off-board DC charging infrastructure, being supplied a DC voltage (between $200 \mathrm{~V}$ and $500 \mathrm{~V}$ ) allowing a maximum of $200 \mathrm{~A}$ with a maximum power level of $100 \mathrm{~kW}$.

There exists however some controversy on the DC charging process control standards existing already a widely used standard, CHAdeMO [2], that specifies the DC fast charger systems up to a maximum power of $62.5 \mathrm{~kW}$.

Despite the divergences considering protocols and connection standards, the development of such high power systems brought to discussion a common concern regarding the capability of the existing electric grids in supporting such power demanding systems and how the grid quality parameters, such as harmonic distortion and the power factor, will be affected. This analysis is particular important considering the higher power charging systems. By being on the lower layers of the electric mobility supply chain, the way they are developed 
plays an important role in the future of electric mobility. The typical topology of such charging system is presented in the figure 1

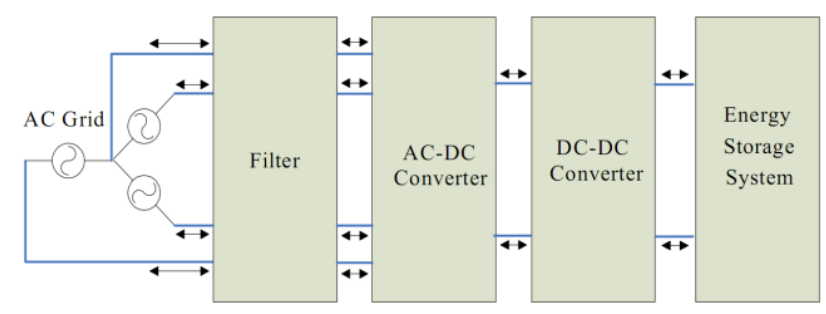

Fig. 1. EV DC charger topology for a three-phase input system.

As is depicted in figure 1, the development of such system embraces several interconnected stages that, individually considered, present complex design challenges. Therefore, the analysis performed in this paper will be segmented into four main sections. The challenges posed to the electric grid in order to support the deployment of such EV's charging network are briefly described in the section 2, highlighting the requirements of the AC-DC rectification stage and its implications on the design of the following system's stage. The DC-DC conversion system, which is the stage with more relevance for the present work, will be analysed in the section 3 . In the section 4 will be presented a modular design approach for implementing a DC-DC converter capable of performing the constant-current / constant-voltage charging of a battery over a broad range of possible load scenarios. Finally, in section 5 a prototype system according to the previously debated topics will be briefly presented.

\section{AC-DC Conversion Stage}

The input stage of the AC-DC converter is the key component for the good performance of the charging system, concerning the grid connection. The implementation to be adopted has to meet the regulatory requirements regarding to the Total Harmonic Distortion (THD) limits and Power Factor Correction (PFC). The simplest topologies of AC-DC rectifiers, typically performed by a diode bridge and a capacitor, draw nonsinusoidal currents from grid, deteriorating severely the power quality of the distribution network. The figure 2 is a good representation of the problem under analysis. In a single phase system, an AC-DC rectifier without PFC draws from the grid a spiked current waveform, which occurs only when the voltage of the capacitor drops under the value of the grid voltage.

This implementation brings severe problems to system's robustness. In present scenario, the components along the power path must present specifications significantly derated in order to withstand large in-rush currents several times larger than the expected nominal values. The use of PFC systems is approach that must be followed to perform the grid voltage rectification, smoothing this huge peak current and charging the capacitor over the time [3].

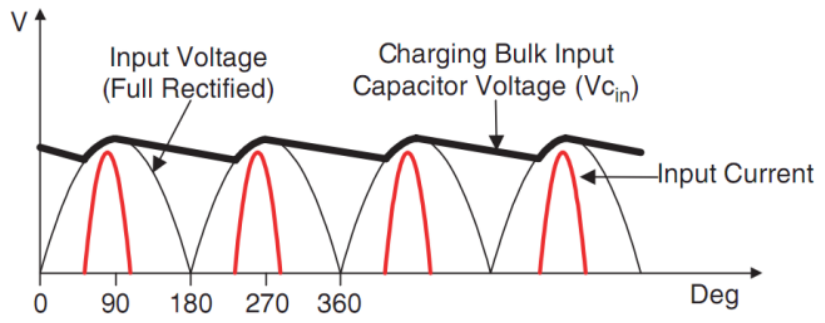

Fig. 2. Voltage and Current waveforms in a simple rectifier circuit.

The generality of PFC circuits are derived from the Boost Switched Mode Power Supply (SMPS) topology due its simplicity and the capability to draw current continuously from the grid. In high power applications, typically greater than $10 \mathrm{~kW}$, six-switch three-phase boost based topologies are preferred [4]. An example of such approach is presented in the figure 3 . In this system, the power factor correction is obtained by controlling the three synchronous rectification legs so the input current of each phase is shaped according to the correspondent phase voltage, leading this way the current to track the voltage phase.

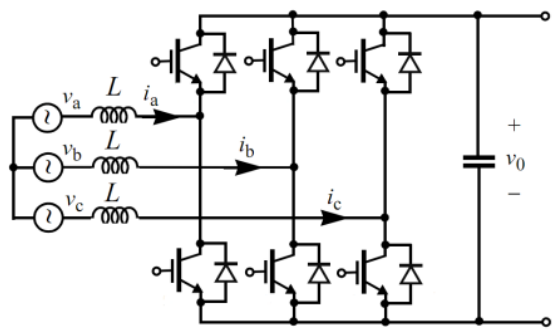

Fig. 3. A six-switch three-phase Boost based PFC.

However, despite the benefits brought by the boost topology based rectification designs to the grid quality parameters, its operation principle inevitably implies the DC bus voltage to be at a significantly higher value than the typical $400 \mathrm{~V}$ peak voltage of an European three-phase grid. It is common to find in the related literature threephase rectification systems with output voltages higher than $700 \mathrm{~V}[5,6,7]$, far superseding the maximum voltage of the currently used battery systems. Therefore, as will be presented in the following section, a DC-DC conversion stage is required to bridge the rectification system with the battery to be charged.

\section{DC-DC conversion stage}

Since the voltage levels provided by the output of the Power Factor Correction stages (AC-DC Conversion) are typically higher than $700 \mathrm{~V}$, stepping-down its value becomes necessary in order to comply with EV battery specifications. This task is typically handled by Switched Mode Power Supplies (SMPS) based on the Buck topology. This DC-DC converter comprises the final stage of the fast-charging system so it may be subjected to non predicted external events that can cause several failure situations. Therefore, it is important for the chosen design to comply with strict security regulations in order to protect the hardware itself and the end user. The major 
draw-back of the Buck topology is the absence of galvanic isolation between its input and output. Since this stage does not provide this feature, the charging system has to use either a line frequency transformer, placed before the AC-DC stage, or a high frequency transformer, placed at the AC-DC stage (typically a Boost SMPS implementing the rectification and Power Factor Correction (PFC)). As the later solution is not commonly available, the adoption of the former one increases significantly the system's final cost since the design of a low frequency transformer for high power applications has higher costs in magnetic materials. As so, when high power density is required and lower costs are mandatory, the galvanic isolation is implemented in a high frequency stage, typically at the DC-DC conversion.

An architecture featuring the cited requirement is the Forward SMPS converter, as is depicted in figure 4. This system can be seen as an isolated buck converter, where the transformer is simply a voltage gain element. The usage of a full bridge to control the current flow through the primary winding optimizes the energy transfer to its secondary side, allowing the use of duty-cycles higher than $50 \%$ and not requiring the use of transformer reset circuitry (as is mandatory in half-bridge based systems [8]) since the core saturation is avoided by applying an alternating polarity voltage across its input.

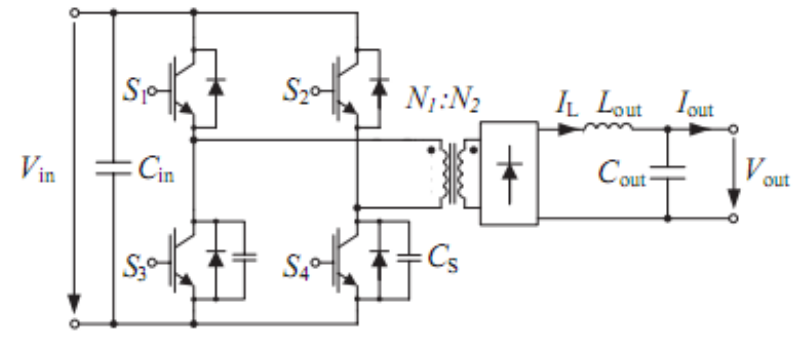

Fig. 4. Forward DC-DC converter with high frequency isolation

As the DC Bus voltage between the AC-DC and the DCDC converters largely supersedes the specifications of most battery systems, the choice of the transformer's turn ratio $\left(\mathrm{N}_{1}: \mathrm{N}_{2}\right)$ is of utmost relevance for performing the required step down stage. On it depends the system's maximum output voltage and the even distribution of the energy flow through the system elements over the switching period to avoid large in-rush current. The dependency of the output voltage and the operational duty-cycle (D [\%]) on the latter parameter is evinced on the control-to-output system's transfer function, presented in the equation (1).

$$
\frac{V_{\text {out }}}{D}=\frac{V_{\text {in }} \cdot N_{1} / N_{2}}{C_{\text {out }} L_{\text {out }} s^{2}+L_{\text {out }} / R_{\text {load }} s+1}
$$

When the output specifications of the system are not expected to vary significantly, the transformer turn ratio is chosen to provide the required voltage step down when the operational duty-cycle is near its $100 \%$ rate. However, since there is currently no standard among manufacturers concerning the used battery systems voltage, optimizing the SMPS design so it can operate under its nominal power over the broad range of possible output voltage is somewhat a difficult task. For example, a SMPS working at its nominal power and optimized for a step-down stage providing a $600 \mathrm{~V}$ output but operated with a reduced duty-cycle in order to control the output voltage of the charger for a $300 \mathrm{~V}$ battery has only approximately $50 \%$ of its switching period available for transferring the required energy along the SMPS power path, what invariably results in a higher current stress on the system's components.

In order to develop a charging system capable of sustaining such situation, two approaches can be followed. The first one consists in designing a single power stage, where the switching elements, the isolation transformer, the inductor and capacitors have to be de-rated for a higher peak power than the nominal one, so they can sustain the overstress caused when supplying its nominal power under low operational duty cycles. This approach may require the use of a high number of paralleled switching elements, in order to reduce the conduction losses, the use of custom bulky inductors, with high inductance and saturation current values, and large output capacitors capable of minimizing the output voltage and current ripple. Nevertheless, there is a clear economic factor that is being disregarded when this approach is considered. The alternative line of work, and the most advantageous one, relies on the use of several miniconverter cells that work all together in order to achieve the overall system demands. Such approach will be presented in the following subsections.

\section{DC-DC converter modular design}

A divide-and-conquer strategy is a commonly used method to address problems of high complexity in a multiplicity of domains. By breaking down the major challenge into several smaller tasks, simpler and more efficient partial solutions can be found for each one of the sub-tasks and, consequently, solve the initial problem. When considering the particular application presented in this work, a modular and re-configurable system composed by several SMPS is a promising approach to develop an efficient charging system capable of operate under nominal power over a broad range of possible load scenarios.

Depending on the type of battery to be charged, the SMPS may be required to supply its nominal power under conditions that demand either for high current / lower voltage or high voltage / lower current configurations. In order to develop a SMPS capable of complying simultaneously with such requirements, the system specification can be decomposed in two stages:

- Develop a single sub-module optimized to provide a fraction of the output voltage/current of the overall charging system.

- Interconnect the output of several sub-modules, either in series or in parallel, in order to achieve the system's maximum power specifications.

While the parallel configuration of several modules favours a design capable of supplying high charging 
currents (while sharing the same output voltage), the serial one increases the voltage output of the charging system (while sharing the same output current). The figure 6 depicts the possible configuration scenarios (considering the existence of two SMPS), where each module is developed according to the forward topology presented previously in the figure 4 . In a real system, many more modules can be used to increase the total output power.

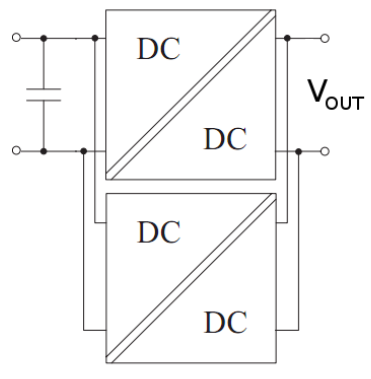

a)

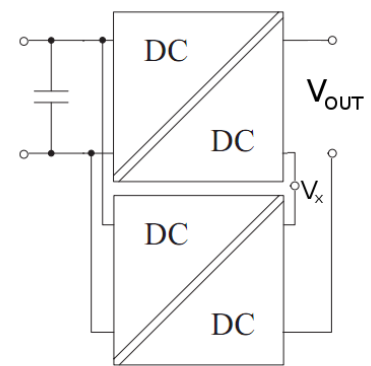

b)
Fig. 5. Two stage Forward converter. a) Parallel output operation, for high current demanding loads b) Serial output operation, for high voltage demanding loads.

In order to maximize the system's global efficiency in the broad range of operation conditions, considering that $n$ DC-DC converters are working cooperatively, each one's design shall be optimized considering the following constrains:

- The maximum output voltage $\left(\mathrm{V}_{\max }\right)$ is limited by the number of modules connected in series when supplying the charging system's maximum output voltage. Therefore, the maximum voltage that each module has to supply is given by the ratio (Max Output Voltage)/ $n$.

- The maximum output current $\left(\mathrm{I}_{\max }\right)$ is limited by the number of modules connected in parallel and the minimum output voltage $\left(\mathrm{V}_{\min }\right)$ from which the SMPS is capable of supplying its nominal power. Therefore the maximum current that each module is capable of supply is given by the ratio $\left(P_{\max }\right) /\left(n * V_{\min }\right)$.

- The system's total output power must be evenly distributed among the $n$ sub-modules.

The referred parameters are depicted in the I/V P/V curves (for a single sub-module), presented in the figure 6.
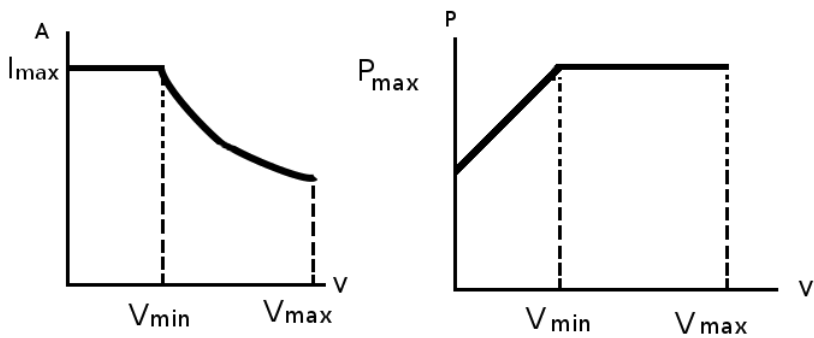

Fig. 6. Expected P/V and A/V curves for a single SMPS.

Despite the system's capability of sustaining its maximum charging current $I_{\max }$ at $V_{\text {min }}$, for higher charging voltages this value is gradually reduced in order to minimize the stress on the switching devices and diodes during the hard-switching transients and therefore achieve a more robust operation of the system.

In the following sub-sections the advantages brought by the system's modular design will be further depicted considering the two possible configurations.

\section{A. Increasing system's maximum output current}

When high current throughput is a requirement, a commonly followed approach is to split the output power stage into several paralleled SMPS. As an immediate consequence, the power specification of each subsystem can be significantly lowered and inherently the switching and conduction stresses sustained by the switching and filtering elements. Furthermore, even in charging situations that demand lower operational duty-cycle, the stress posed by the inrush current is reduced since the power that each device has to supply is lower, when comparing with a single system design. These benefits are particularly highlighted in the works of [9] and [10], where the operation of three interleaved buck converters based on half bridge topology is evaluated in applications that handle high output currents. Such topology is presented in the figure 7 .

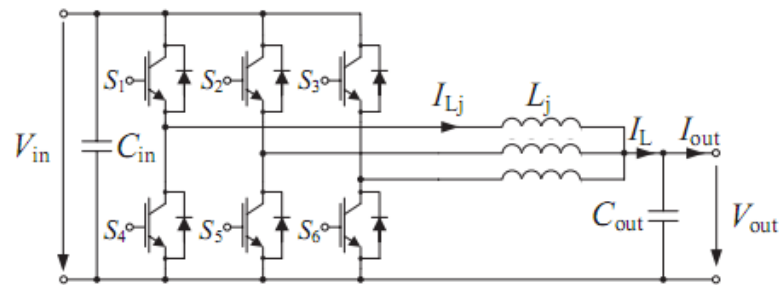

Fig. 7. Interleaved Buck converter [9].

The same configuration principle is also found in SMPS based on the forward converter, as in the works of [11], scaling the current throughput of the DC-DC converter while maintaining the galvanic isolation capability. Apart from improving the power rating, paralleling several modules leads to improved output current and voltage regulation during the constant current $(\mathrm{CC})$ and constant voltage (CV) charging periods. This is of particular importance in the considered application scenario as high current/voltage ripple is responsible for an accelerated degradation of the battery life time due to overheating of its cells (caused by high frequency loss mechanisms) [12]. By introducing a phase delay in the control of each paralleled buck converter, their operation becomes interleaved and current ripple cancellation can be obtained at the output of the system. This eases the design of the filtering stage (both inductor and capacitor) since higher current ripple is admitted for each sub-circuit. Similar results on the output regulation could be obtained by simply increasing the switching frequency of the DC-DC converter by the number of interleaved circuits. However, considering the expected power levels of his application, the choice of the switching elements' specifications, such as rise/fall times, reverse recovery time and forward conducting resistance, may become critical for the 
converter's proper operation and minimization switching and conducting losses.

Figure 8 depicts a particular scenario, where the operation of three interleaved buck converters is shifted $120^{\circ}$ from each other, so maximum ripple cancellation is obtained. This approach is easily scalable so, by increasing the number of paralleled sub-modules and adjust their relative control phase, the system's current throughput and its maximum ripple can be further improved.

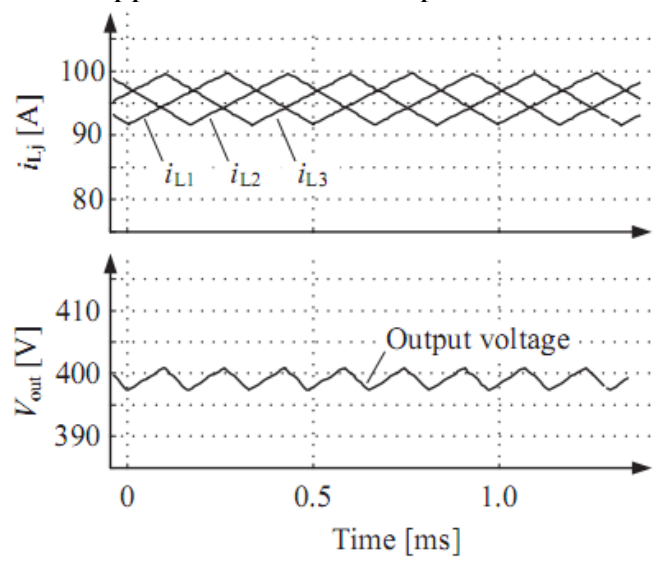

Fig. 8. Three paralleled buck converters output current waveforms, which operation is interleaved so the total output voltage ripple is reduced [9].

\section{B. Increasing system's maximum output voltage}

Since each of the system's DC-DC converter was developed for a fraction of the maximum output voltage, when the sought charging voltage cannot be supplied by a single DC-DC converter the system's modular design can be exploited by connecting in series several modules and equally split the supplied voltage among them. This arrangement is possible since each SMPS output is floating due to existence galvanic isolation from their input. Even though the later configuration is hardly a novel concept, it has been scarcely addressed by the published literature. However, the use of this approach for scaling the advantages of the converter presented in the section 4 is nothing but a natural consequence of the modular design flexibility, reducing the high voltage/high current stress by several sub-systems.

The system's versatility requires a tight control of each converter output in order to evenly distribute the supplied power by the available sub-systems. Component tolerances drive imbalances and timing errors are among the possible disturbances that may result in an uneven power distribution and consequently drift the system from its optimal operation point. This problem is particular important at the constant-current charging stage, during which the system works closer to its nominal power specification. Therefore, in order to avoid such imbalances, the control system of each converter requires measurements regarding its own voltage and current output but also concerning the overall system output, so its respective control set-points are determined considering the demanded fraction of supplied power.
While the detection of system imbalances under constantcurrent charging mode seems trivial when opting by the paralleled operation of the modules, since the same voltage is imposed by the battery to each converter's output and it is possible to independently measure each one's supplied current, when several modules are connected in series the same cannot be said. Considering the example depicted in the figure 5-b it is clear that, under constant current charging mode, an even distribution of the transferred power among the available DC-DC Converters is only guaranteed if the intermediate point voltage $\left(\mathrm{V}_{\mathrm{x}}\right)$ is half of the output voltage $\left(\mathrm{V}_{\text {out }}\right)$. In the generic case of having $n$ converters connected in series, each interconnection must present a voltage that is a fraction of $\mathrm{V}_{\text {out }}$.

In order to assure the referred condition, with an arbitrary number of modules connected in series just a single DCDC converter can work on constant current mode, which inherently will set the current that every other device in series provides. The remaining converters must operate under constant voltage output, maintaining a voltage differential of $\mathrm{V}_{\text {out }} / \mathrm{n}$ across their output, guaranteeing this way that the same power is supplied by every module. During the charging procedure, the battery voltage increases gradually, implying this way a constant monitoring of the set-point of the modules operating under voltage feedback.

\section{Experimental results}

In order to assess the proposed configuration, a prototype system composed by two sub-modules was developed, as is depicted in figure 10 .

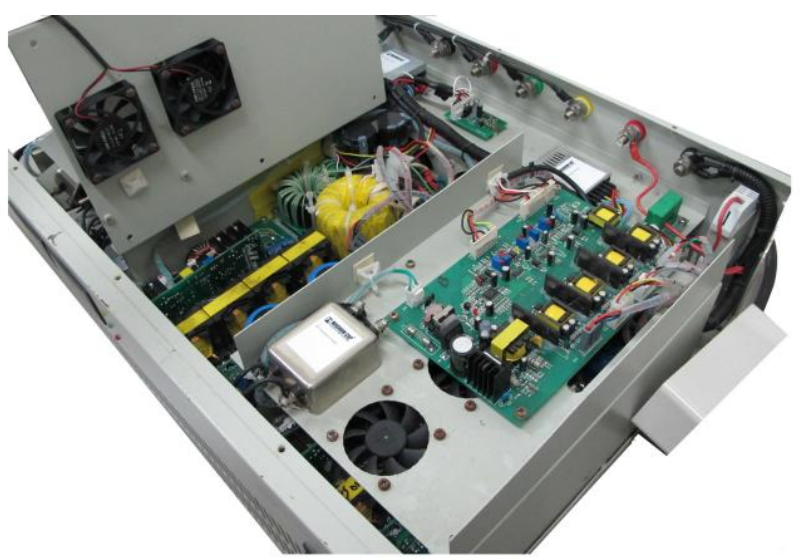

Fig. 10. Developed prototype system, comprising two $5 \mathrm{KW}$ sub-modules.

The considered charging system was specified to supply a maximum of $10 \mathrm{KW}$, operating under a $150 \mathrm{~V}-600 \mathrm{~V}$ range. Therefore according to the assumptions presented previously in the section 4 each sub-module must be designed according to the following specifications:

- The maximum output power is $5 \mathrm{KW}$

- The maximum output voltage of each SMPS is $300 \mathrm{~V}$ 
- The maximum output current is $33 \mathrm{~A}$ under a $150 \mathrm{~V}$ load

When charging a battery pack under $300 \mathrm{~V}$, the two SMPS work in parallel, with a maximum output current of $33 \mathrm{~A}$ each. A load composed by batteries from $300 \mathrm{~V}$ up to $600 \mathrm{~V}$ would require the connection the two available submodules' output in series with a maximum output current of $16.5 \mathrm{~A}$ at $600 \mathrm{~V}$.

Since each sub-module is specified to supply its rated power with loads as low as $150 \mathrm{~V}$, there is no reduction on the power supplied by the charging system when its operation is near the $300 \mathrm{~V}$ output voltage. However, there is a significant increase on the current supplied by each sub-system (from $16.5 \mathrm{~A}$ at $300 \mathrm{~V}$ to $33 \mathrm{~A}$ each at $150 \mathrm{~V}$ ) near the point where the system's configuration changes from parallel to serial one. The expected $\mathrm{P} / \mathrm{V}$ and $\mathrm{I} / \mathrm{V}$ curves of the developed system are presented in the figure 11.
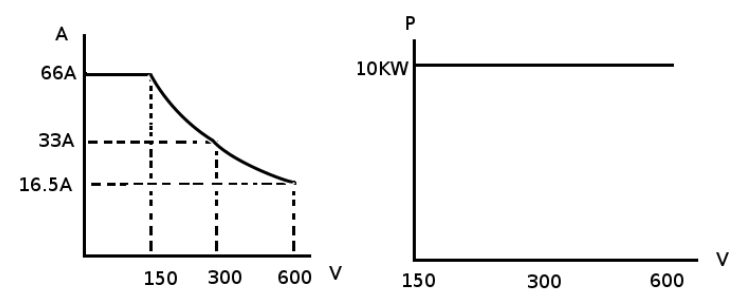

Fig. 11. Expected P/V and I/V curves of the implemented charging system.

In order to assess the operation of the system, four lead battery banks of $200 \mathrm{~V} 7 \mathrm{Ah}$ were assembled. These loads allowed to test the system under $200 \mathrm{~V}$ load and $400 \mathrm{~V}$, testing the parallel and series configuration of the system respectively. Under constant current operation, with parallel configuration and all the battery strings connected in parallel, a charging current of $28 \mathrm{~A}$ was used being observed a current ripple of approximately 1.2A. With the two SMPS connected in series and the battery strings reorganized in order to create a $400 \mathrm{~V} 14 \mathrm{Ah}$ battery, under a constant charging current of 14A the measured ripple was approximately doubled, being measured 2.1A. The results were obtained by using a Tektronix A6222 isolated current and a Fluke 289 True-RMS multimeter. The power levels of the referred test scenario were limited to $5.6 \mathrm{~kW}$ due to limitations of the load used. For better validation of the developed prototype, the system's load has to be improved in order to sustain higher delivered power levels.

\section{Conclusions}

This paper reviewed some of the major concerns when developing high power charging systems and proposed a system easily adaptable to the broad diversity of specifications of the upcoming generations of electric vehicles. Modularity seems to be the key for the success of the overall system, since it allows the use of medium power devices (already well studied in the literature) to implement efficient systems with higher power capabilities and, ultimately, reduce the EV batteries charging times. The flexibility of the proposed system also overcomes the indefinition regarding the maximum power specifications of the charging protocols.

\section{Acknowledgement}

This work was partially supported by MagnumCap - Electrical Power Solutions ( www.magnumcap.com ) and by FCT Foundation for Science and Technology in the context of the project FCOMP-01-0124-FEDER-022682 (FCT Reference PEstC/EEI/UI0127/2011)

\section{References}

[1] SAE Electric Vehicle and Plug in Hybrid Electric Vehicle Conductive Charger Couple, Standard J1772, standards.sae.org/j1772_201210/, Accesses at 24-Oct2012.

[2] "CHAdeMO Association", www.chademo.com, Accessed at 24-Oct-2012.

[3] "Fairchild Application Note 42047 Power Factor Correction (PFC) Basics".

[4] O. Onar D. Erb and A. Khaligh, "Bi-Directional Charging Topologies for Plug-in Hybrid Vehicles", IEEE Bucharest Power Tech Conference, 2009.

[5] C Zou K. Yao, X. Ruan and Z. Ye, "Three-phase SingleSwitch Boost PFC Converter with High Input Power Factor", Energy Conversion Congress and Exposition (ECCE), 2010.

[6] F. Lee R. Zang, "Optimum PWM Pattern for a ThreePhase Boost DCM PFC Rectifier", Applied Power Electronics Conference and Exposition, 1997.

[7] J. Kollar and F. Zach, "A Novel Three-Phase Utility Interface Minimizing Line Current Harmonics of HighPower Telecommunications Rectifier Modules", IEEE Transactions on Industrial Electronics, vol.44, no. 4, 1997.

[8] Ki-Bum Park, "Interleaved Forward Converter for High Input Voltage Application with Common Active-Clamp Circuit and Series Rectifier", Twenty-Fourth Annual IEEE Applied Power Electronics Conference and Exposition, 2009.

[9] H. Zelaya-De La Parra A. Coccia N. Butcher D. Aggeler, F. canales and O. Apeldoorn, "Ultra-Fast DC-Charge Infrastructure for EV-Mobility and Future Smart Grids", Innovative Smart Grid Technologies Conference Europe, 2010.

[10] O. Onar D. Erb and A. Khaligh, "Automotive DC-DC Bidirectional Converter Made with Many Interleaved Buck Stages", IEEE Transactions on Power Electronics, vol.21, 2006.

[11] K. Zhang T. Jin, K. Zhang and K. Smedley, "A New Interleaved Series Input Parallel Output (ISIPO) Forward Converter With Inherent Demagnetizing Features", IEEE Transactions on Power Electronics, Vol. 23, no. 2, 2008.

[12 C. Chen J. Wang, K. Zou and L. Chen, "A High Frequency Battery Model for Current Ripple Analysis", 25th Annual IEEE Applied Power Electronics Conference and Exposition, 2010.

[13] R. W. Erickson and D. Maksimovic, Fundamentals of Power Electronics, Kluwer Academic Publishers, 2000.

[14] D. Beatty and I. Batarseh, "Topical Overview of SoftSwitching PWM High Frequency Converters", Electrical and Computer Engineering Department University of Central

Florida. 